\title{
A saúde mental nos estudos sociais da ciência: apontamentos sobre a produção de conhecimento
}

\author{
Alana de Paiva Nogueira Fornereto Gozzi ${ }^{a}$, Edson Malvezzi ${ }^{\mathrm{b}}$, Meliza Cristina da Silva ${ }^{\mathrm{b}}$, \\ Márcia Niituma Ogata ${ }^{c}$ \\ aDepartamento de Terapia Ocupacional, Universidade Federal de São Carlos - UFSCar, São Carlos, SP, Brasil. \\ bUniversidade Federal de São Carlos - UFSCar, São Carlos, SP, Brasil. \\ 'Departamento de Enfermagem, Universidade Federal de São Carlos - UFSCar, São Carlos, SP, Brasil.
}

\begin{abstract}
Resumo: Os problemas mentais e comportamentais exercem um considerável impacto sobre os indivíduos, suas famílias e as comunidades onde vivem. Por esse motivo, a saúde mental tem sido alvo de diversas pesquisas recentes, dada sua complexidade, importância da discussão e aumento da incidência dessas patologias no mundo. Uma em cada quatro famílias tem pelo menos um membro que apresenta um problema mental ou comportamental. Cabe, dessa forma, a verificação de existência da temática no campo de Estudos Sociais da Ciência e como o tema tem sido abordado. Este estudo tem como objetivo a realização de uma revisão bibliográfica crítica da literatura, retrospectiva, em forma de ensaio, norteada por uma prerrogativa que envolve as peculiaridades apontadas pela literatura sobre saúde mental no campo da Ciência, Tecnologia e Sociedade (CTS). O ensaio discute as tendências e linhas de investigação em curso, relacionando saúde mental e CTS e pode indicar lacunas ainda existentes. Espera-se que este trabalho possa trazer contribuições e revele a importância da discussão sobre a saúde mental no campo social, assim como a forma com a qual temática vem sendo abordada sob diferentes olhares.
\end{abstract}

Palavras-chave: Saúde Mental, Ciência, Tecnologia e Sociedade, Revisão do Estado da Arte.

\section{Mental health in social studies of science: notes about knowledge production}

\begin{abstract}
Mental problems have considerable impact on individuals, on families and on the communities where they live. For this reason, mental health has been the subject of several recent surveys, given its complexity, emergent discussion and increased incidence of mental disorders in the world. The investigation of the scientific/international production and knowledge about the connection of this theme with the field of Science, Technology and Society (STS) is, therefore, very timely. This study aims to carry out an integrative and retrospective literature review guided by a prerogative involving the peculiarities of mental health in the STS field as presented in the international literature. The review discusses the trends and ongoing research lines, linking mental health and STS and indicating possible gaps. It is hoped that this work brings contributions and reveal the importance of discussing mental health in the STS field.
\end{abstract}

Keywords: Mental Health, Social Science and Technology, Review Literature as Topic.

Autor para correspondência: Alana de Paiva Nogueira Fornereto Gozzi, Departamento de Terapia Ocupacional, Universidade Federal de São Carlos, Rodovia Washington Luiz, Km 235, Monjolinho, CEP 13565-905, São Carlos, SP, Brasil, e-mail: alanafornereto@gmail.com Recebido em Ago. 18, 2016; 1ª Revisão em Fev. 1, 2017; Aceito em Fev. 21, 2017. 


\section{Introdução}

Os problemas mentais e comportamentais exercem um considerável impacto sobre os indivíduos, suas famílias e as comunidades onde vivem. Por esse motivo, a saúde mental tem sido alvo de diversas pesquisas recentes, dada sua complexidade, emergência de discussão e aumento da incidência de transtornos mentais no mundo. Uma em cada quatro famílias tem pelo menos um membro que apresenta um problema mental ou comportamental, dado que reafirma a necessidade dessa discussão (ORGANIZAÇÃO..., 2002).

A saúde mental é um campo interdisciplinar, tendo em vista as inúmeras complexidades com as quais deve lidar. A limitação por disciplinas para o campo não é o suficiente para a gestáo da clínica das mais diferentes problemáticas de saúde mental.

Os transtornos mentais têm forte impacto na saúde pública, não sendo exclusividade do Brasil. Segundo Thornicroft e Tansella (2010), em países menos desenvolvidos, o número de pessoas com esquizofrenia aumentou de 16.700 milhóes em 1985 para 24.400 milhóes em 2000.

Os transtornos mentais, tendo em vista a importante interferência na vida cotidiana das pessoas, na participação social e na expectativa de vida, revelam consequências importantes para indivíduos que os manifestam e também para suas famílias e cuidadores que acabam se responsabilizando pelo cuidado. A tarefa do cuidado em saúde mental produz uma carga, exigindo dedicação de quem cuida, o que acaba por produzir uma redução da produtividade econômica e da geração de renda desses familiares.

Para os indivíduos com transtornos mentais, as consequências são severas:

[...] sofrimento causado pelos sintomas, a baixa qualidade de vida, a perda da independência e da capacidade de trabalho e uma integração social deficiente (THORNICROFT; TANSELLA, 2010, p. 10).

Para que esses sujeitos com acometimentos na saúde mental sejam assistidos, considerando inclusive essas informaçôes epidemiológicas, há que disponibilizar intervençóes acessíveis e tecnologias em saúde adequadas pautadas na complexidade de suas necessidades. Pensando nessa oferta de serviços e açôes de cuidado, assim como na forma como a saúde mental tem evoluído ao longo dos tempos, carregando consigo um estigma expressivo, o campo da Ciência, Tecnologia e Sociedade (CTS) pode auxiliar nessa compreensão. Oferecendo ferramentas e aportes para uma discussão qualificada, pautada nas necessidades, em inovaçóes tecnológicas e que seja para e na comunidade de fato, trazendo benefícios e garantindo acesso.

\section{CTS: um novo paradigma}

Vive-se um século de intensas modificações e progressos, como nunca se vira. Este movimento muito se deve aos grandes cientistas, que alimentaram a vida com suas descobertas, principalmente entre os séculos XVIII eXX. Esse modelo de desenvolvimento traz na ciência uma grande esperança para a humanidade de melhora na qualidade de vida (ZAUITH et al., 2011; SANTOS, 1987).

Este processo evidencia certa ambiguidade e complexidade, que naturalmente reflete no presente e no futuro desse desenvolvimento que estamos envolvidos. Nada mais requer do que simples perguntas que, afinal, movem o ser humano a compreender a natureza à sua volta e compreender-se enquanto ser no mundo.

Jean-Jacques Rousseau, filósofo, teórico político, escritor e compositor do século XVIII, foi um dos que elaborou uma dessas simples perguntas que pode esquentar esse debate: $O$ progresso das ciências e das artes contribuirá para purificar ou para corromper os nossos costumes? (SANTOS, 1987). Seu questionamento leva a pensar sobre esse desenvolvimento que os séculos XX e XXI têm proporcionado e também quem são as pessoas que se beneficiam desse desenvolvimento. Assim como pensar na contribuição positiva ou negativa dessa ciência para nossa felicidade, como ressalta o autor citado.

As condiçôes sociológicas e psicológicas são diferentes no decorrer dos tempos e mais complexas em nossa época. Por esse motivo, o movimento de produção de conhecimento deve acompanhar essas transiçóes e adaptaçóes, respondendo às necessidades reais da sociedade que acompanha esse processo.

O modelo da racionalidade é o paradigma dominante na produção do conhecimento científico, com o predomínio das ciências naturais do séc. XVI. Esta ciência moderna desconfia das evidências e experiências do senso comum, presentes no saber aristotélico e medieval, trazendo um rigor científico importante e predominante neste fazer (SANTOS, 1987). Para Zauith et al. (2011), essa forma vem sendo revista, já que têm sido colocada à prova as concepçôes sociais, políticas, econômicas e culturais presentes nesse movimento.

A perspectiva da ciência moderna se preocupa com a causalidade dos fatos e funcionamento das coisas, com uma tendência a dominar e transformar o mundo e a natureza. Esse movimento, na época, 
influenciou o estudo da sociedade, alvo também a ser compreendido, caracterizando-se como uma ciência empírica aos moldes das ciências naturais.

Pôde-se visualizar que esse modo, com certa prioridade cognitiva das ciências naturais, não possibilitava a compreensão da ação da humana, que é mais subjetiva e social do que se propunham as metodologias adotadas. Assim, uma crise do paradigma dominante emerge, clamando pelo reconhecimento de que as condiçóes sociais exigiam outras formas de pesquisa, um lado $\mathrm{B}$, como discutem Zauith et al. (2011).

O rigor científico moderno e modelo linear de desenvolvimento da ciência é questionado nesse contexto, evidenciando um declínio da supremacia da causalidade, tendo em vista os aspectos humanos que se mostram relevantes para sociedade. Nesse sentido, é possível verificar a eminência de um campo que se preocupasse com aspectos relevantes, para além das grandes descobertas científicas.

Nesse cenário, um paradigma emergente, então, acaba por prezar por um conhecimento prudente para uma vida decente: um paradigma social, onde não mais vemos distinção clara entre as ciências naturais e sociais (SANTOS, 1987).

Cabe, dessa forma, a verificação de existência da temática "saúde mental" no campo CTS, e como o tema tem sido abordado. Essa articulaçáo se dá a partir de uma concepção de CTS, regulada pela relação entre produção e circulação do conhecimento na sociedade, ou seja, na perspectiva de mudança da centralidade do poder científico, da ciência e da construção de conhecimentos voltadas para os interesses da sociedade (do sujeito e coletivo). Nesse caso, os problemas de saúde mental que vêm em um tom crescente no mundo.

Este trabalho tem como objetivo abrir um debate sobre a forma de abordagem do tema "saúde mental" no campo dos Estudos Sociais da Ciência, fazendo uso de artigos específicos da área, de forma a também aproximar a discussão para Brasil.

\section{Método}

O presente trabalho se constitui em um ensaio crítico. Para subsidiar a discussão, foi realizada uma revisão integrativa da literatura (MENDES; SILVEIRA; GALVÃO, 2008) para auxiliar o debate a respeito das peculiaridades apontadas pela literatura sobre saúde mental no campo CTS. A escolha por este tema vem da intenção de conhecer o que já foi produzido a respeito, as discussóes fomentadas e algumas metodologias utilizadas nos artigos científicos referentes ao assunto, assim como a relação estabelecida pelos autores com campo CTS. Uma hipótese inicial girava em torno da não existência desse tipo de estudo no campo dos Estudos Sociais da Ciência.

A estratégia de busca aos periódicos ocorreu no mês de outubro/2014, utilizando os periódicos da área indexados no banco de dados Web of Science. As palavras-chave utilizadas foram mental health.

Após esse levantamento dos artigos, foi elaborada uma ficha para anotação de dados que os textos evidenciavam, estruturando a análise posterior dos artigos levantados. Este instrumento continha as seguintes informaçôes: nome do artigo, delineamento metodológico, principais resultados, periódico e ano de publicação. Esta estratégia de sistematização dos dados foi baseada no trabalho publicado por Iglesias e Avellar (2014).

A análise dos artigos foi realizada a partir da técnica da análise de conteúdo (BARDIN, 2009). Utilizou-se a técnica de análise temática, o que possibilitou a identificaçáo de algumas categorias de análise, por meio da elaboração de núcleos de sentido, extraídos da leitura dos artigos.

No banco de dados em questão foram inicialmente localizadas as revistas da área CTS, dentre elas: Arbor; Bulletin of Science, Technology and Society; Research Evaluation; Science Communication; Social Studies of Science; Science, Technology and Human Values; Science, Technology and Society.

Foram localizados um total de 9 artigos, utilizando-se da palavra-chave mental health para a busca em cada um dos periódicos. Em apenas uma das revistas selecionadas (Science, Technology and Society) não foi localizada nenhuma publicaçấo com as palavras-chave indicadas. O periódico Bulletin of Science, Technology and Society não disponibiliza seus artigos online, por esse motivo foram descartados. Por fim, utilizaram-se 7 artigos. O critério de inclusão envolveu a localização dos artigos que apresentassem as palavras-chave usadas em meio eletrônico.

Após a seleção dos artigos, os mesmos foram lidos na íntegra e deles extraídos pontos-chave, principais discussóes e categorias de análise, assim como possíveis relaçóes entre saúde mental e CTS.

\section{Resultados e Discussão}

$\mathrm{Na}$ Tabela 1, os artigos incluídos na revisão são apresentados a partir do nome dos autores, ano de publicação, nome do periódico e nome do artigo. 
Tabela 1. Artigos incluídos na revisão.

\begin{tabular}{ccl}
\hline $\begin{array}{c}\text { Autores (ano da } \\
\text { publicação) }\end{array}$ & Periódico & \multicolumn{1}{c}{ Nome do artigo } \\
\hline Lewison et al. (2004) & Research Evaluation & $\begin{array}{l}\text { Outputs and expenditures on health } \\
\text { research in eight disease areas using a } \\
\text { bibliometric approach, 1996-2001. }\end{array}$ \\
\hline Pols (2006) & Science Technology Human Values & $\begin{array}{l}\text { Accounting and washing: good care in } \\
\text { long-term psychiatry. }\end{array}$ \\
\hline Armstrong (2009) & Social Studies of Science & $\begin{array}{l}\text { Origins of the problem of health-related } \\
\text { behaviours: a genealogical study. }\end{array}$ \\
\hline Kendall-Taylor (2012) & Science Communication, & $\begin{array}{l}\text { Conflicting models of mind: mapping } \\
\text { the gaps between expert and public } \\
\text { understandings of child mental health. }\end{array}$ \\
\hline Palomo (2013) & Arbor & $\begin{array}{l}\text { Locos entre cuerdos, cuerdos en ambientes } \\
\text { patológicos. }\end{array}$ \\
\hline Pickersgill (2013) & Social Studies of Science & $\begin{array}{l}\text { How personality became treatable: } \\
\text { The mutual constitution of clinical } \\
\text { knowledge and mental health law. }\end{array}$ \\
\hline Weber e Backer (2013) & Science Communication & $\begin{array}{l}\text { Science-based communication strategy for } \\
\text { a Federal Health Agency. }\end{array}$ \\
\hline
\end{tabular}

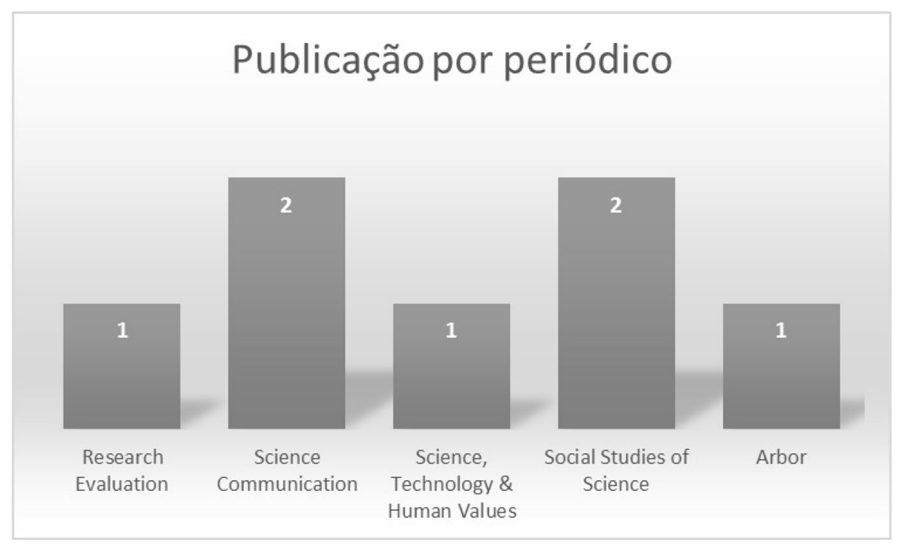

Figura 1. Distribuição das publicações por periódicos.

A análise bibliométrica permitiu a exposição de dados quantitativos sobre as buscas, como aprofundado em seguida. Com relação ao ano de publicação dos artigos encontrados, houve uma concentração no ano de 2012, com 3 publicações, sendo o mais antigo de 2004 e o mais recente de 2013. Apenas um dos artigos incluídos em língua espanhola, sendo os demais em língua inglesa.

Dentre os periódicos encontrados, Science Communication e Social Studies of Science tiveram ambos duas publicações, e os demais apresentaram um artigo cada um, como evidencia o gráfico (Figura 1).

Observando o país de origem das publicaçôes, vemos uma concentração das publicações na Inglaterra e, em segundo lugar, os Estados Unidos
(Figura 2). Esta concentração pode ser confirmada pelo país de origem do periódico.

A leitura dos artigos encontrados permitiu a identificação de algumas categorias referentes à saúde mental e a forma como os autores nas revistas específicas do campo CTS aprofundam a discussão de tais temas a partir dessa abordagem. Tais temas serão aprofundados em seguida e discutidos também com a inclusão de outros achados científicos, além do que fora extraído dos artigos da revisão.

Podemos elencar as seguintes categorias: Pesquisa em saúde mental e gastos; Difusão científica (populaçáo, clínica e políticas); Descompasso entre ciência (pesquisa) e clínica/ políticas; Ciência como missão de promover o bem público; Tecnologias leves e relacionais para saúde mental; e Institucionalização da loucura e suas consequências. 


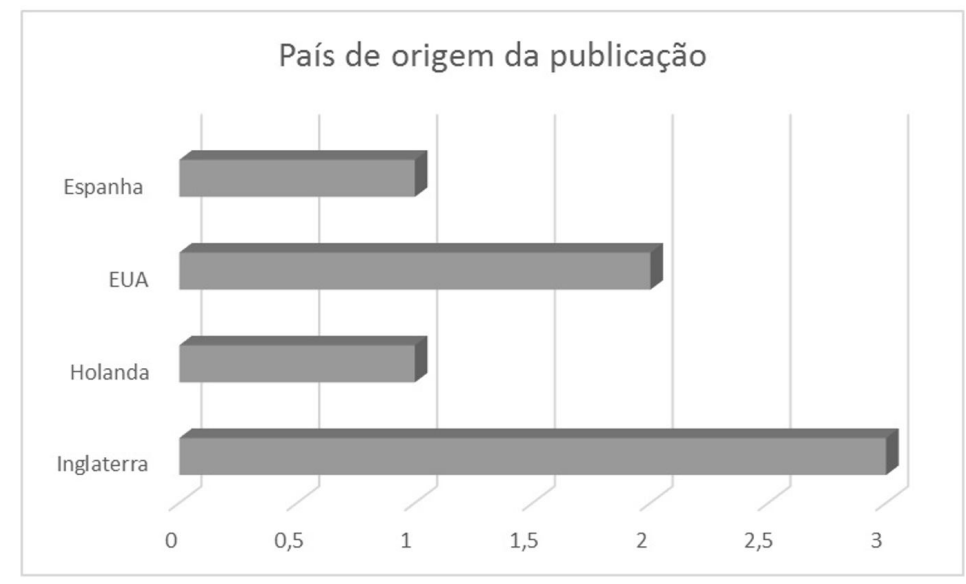

Figura 2. País de origem da publicação.

\subsection{Pesquisa em saúde mental e gastos}

A pesquisa em saúde mental tem entrado na agenda de prioridades de pesquisa em diversos países. Não apenas por dados da OMS (ORGANIZAÇÃO..., 2002) sobre alto índice de incidência de problemas mentais no mundo, mas também pela necessidade de formulação de políticas públicas que possam abordar esse problema de frente. Tal fato é confirmado ao encontrarem-se artigos com a temática saúde mental em periódicos de outro campo, como CTS. Um dos artigos evidencia a entrada (e o jogo) das indústrias farmacêuticas nas pesquisas em saúde mental e seu largo financiamento (LEWISON et al., 2004). Porém, relacionada ainda com outro dos temas levantados (das Tecnologias relacionais), não se pode negar a importância da descoberta dos fármacos para a psiquiatria, mas não é somente com medicação que a clínica nessa área se desenvolve.

No Brasil, as necessidades da população brasileira determinaram algumas áreas denominadas como estratégicas e que fazem parte da Agenda Nacional de Prioridades de Pesquisa em Saúde, a saber: doenças negligenciadas, doenças não transmissíveis, saúde da mulher e da criança, saúde mental, redução da violência e acidentes, complexo produtivo da saúde, entre outras (BRASIL, 2008). Na saúde mental, são priorizados: cultura e sociedade; compreensão dos agravos em saúde mental; organizaçáo e avaliação de políticas e programas; avaliação, desenvolvimento e aplicação de tecnologias em saúde mental.

A identificação dessa categoria após a leitura dos artigos nos permite afirmar que a saúde mental tem feito parte das discussóes no campo CTS, mostrando não apenas a preocupação dos autores com a temática, mas a emergência de novas pesquisas que tragam tecnologias e inovação que efetivamente possam interferir positivamente no processo de cuidado e compreensão da psiquiatria na sociedade.

\subsection{Difusão científica (população, clínica e políticas)}

A difusão científica, forte eixo da sociologia da ciência, aparece também nessa discussão como tema prevalente. A necessidade de aproximação entre ciência e público em geral, melhorando o acesso à informação, pode ser identificado como uma das funçōes da difusão científica à população.

$\mathrm{Na}$ análise, foi possível identificar que os estudos se preocupam com a forma como as informaçốes sobre saúde mental e uso de substâncias psicoativas estarão disponíveis às pessoas em geral, podendo qualificar a forma como lidam com tais temas (WEBER; BACKER, 2013). Diminuir essa distância entre o conhecimento construído e as pessoas, tornando-o de acesso ao público, pode ser uma forma de enfrentamento dos problemas. Latour (2011) sugere que as caixas-pretas sejam abertas, de forma que todos os que estão de fora possam dar uma "olhadela". A perspectiva da difusão científica é exatamente esta: tornar as informaçóes de acesso livre, para que possam ser utilizadas pela população e para seu desenvolvimento.

Da mesma forma, os artigos internacionais desta revisão ressaltam a necessidade que a difusão científica tem em influenciar o exercício da clínica dos profissionais de saúde mental e a formulação de políticas. A reflexão sobre a prática profissional e a apreensão dos novos conhecimentos construídos pode melhorar o cuidado efetivo dispensado aos pacientes, renovando antigos hábitos de cuidado a partir de pesquisas e novos caminhos. 
A difusão científica também é uma importante estratégia para enfrentamento do estigma da saúde mental em nossos tempos. Em nossa opiniāo, a população e profissionais de saúde mais informados e munidos de ferramentas podem compreender e cuidar melhor das pessoas em sofrimento mental.

\subsection{Descompasso entre ciência (pesquisa) e clínica/políticas}

Ainda nessa perspectiva, o descompasso entre ciência (pesquisa) e clínica/políticas corrobora com essa abordagem. Segundo os autores, ainda existe um hiato entre o que a ciência vem construindo, no que diz respeito a novos conhecimentos em saúde mental, e como eles efetivamente têm incentivado o planejamento tanto da clínica realizada nos serviços como da formulação de políticas públicas. Não sendo exclusividade do Brasil, é urgente a ultrapassagem das discrepâncias entre os preceitos e exercício, como sinaliza Pinto (2012), e outros países também sofrem com essa dificuldade. Um dos objetivos do CTS é exatamente trabalhar para que essa barreira possa ser minimizada.

Kendall-Taylor (2012) e Weber e Backer (2013) expressam um descompasso entre o que é gerado de conhecimento clínico em saúde mental e sua apreensão no dia a dia do trabalho em saúde, do planejamento de políticas públicas e na forma como a população compreende essa temática. Sabemos que ainda existe um estigma importante com relaçáo ao tema, apesar de toda sua ressonância. Uma das consequências dessa estratégia é diminuir essa visão estereotipada de pessoas com transtornos mentais por meio da criação de novos caminhos para trazer a informaçáo e o conhecimento de acesso ao público, ampliando assim a compreensão da sociedade sobre o tema.

Ainda que aparentemente negado, alguns dos números são alarmantes e sinalizam cada vez mais uma ampliação da incidência. Por isso, a difusão científica pode ajudar nesse movimento de valorizar esse campo e sensibilizar as pessoas não somente para tratamento, mas também para promoção de saúde e prevenção de agravos, na opinião dos autores.

\subsection{Ciência como missão de promover o bem público}

Tendo em vista o papel da ciência com a missão de promover o bem público, ou seja, oferecer subsídios para o desenvolvimento da sociedade, nesse caso deve estar relacionada ao atendimento de pessoas com problemas de saúde mental. A análise dos dados nos permite inferir que os autores desses artigos de saúde mental preocupam-se com a forma como os conhecimentos descobertos nessa área realmente contribuam para a melhora desses pacientes, cuidado de acesso às pessoas.

Para Kendall-Taylor (2012), o que é problematizado é uma forma de tornar a ciência mais eficaz em sua missão de promover o bem público, melhorar a tradução científica e a adoção dos conhecimentos na clínica de saúde mental junto aos sujeitos. Ao longo do tempo, o conhecimento acerca do doente mental foi evoluindo e, a cada nova abordagem descoberta, se reconstrói e se consolida enquanto disciplina. E até modificar paradigmas e procedimentos estruturados da clínica, como os adotados nos distúrbios de comportamentos descritos por Armstrong (2009), que em dado momento, se modificaram a partir de novas descobertas.

O bem público que se almeja nesse cenário apresentado no ensaio é o de uma compreensão da sociedade acerca da saúde mental como um problema presente e importante que merece atenção; uma oferta de serviços de cuidado baseado nas necessidades dos usuários e coerente com políticas públicas sensíveis a esses indicadores e descobertas de inovaçóes cada vez mais interessantes para que esse cuidado aconteça, com desenvolvimento de tecnologias, como veremos a seguir.

\subsection{Tecnologias leves e relacionais para saúde mental}

O desenvolvimento de fármacos psicotrópicos foi uma grande inovação para a psiquiatria, no que diz respeito ao manejo dos pacientes. Antes dessas descobertas, a exclusão da sociedade era a forma como se pretendia "tratar" tais sujeitos nos grandes manicômios e asilos, de forma a manter a ordem social, sem aqueles que a pudessem perturbar.

$\mathrm{O}$ uso de tecnologias duras, como equipamentos e medicaçóes, é necessário e importante. Grande parte das pesquisas em saúde, especificamente em saúde mental, se dedicam às descobertas de remédios, e a partir da conceituação de Merhy (2000) se define como tecnologia dura.

Na saúde mental, além desse tipo de tecnologia, espera-se que o profissional de saúde seja capaz de utilizar tecnologias leve-duras, aquelas relacionadas aos saberes profissionais, e também as tecnologias leves, ou seja, relacionais entre trabalhador e usuário (MERHY, 2000). Thornicroft e Tansella (2010) afirmam que, a partir da experiência inglesa, 
mais do que em outras áreas da medicina, a saúde mental depende mais de recursos humanos do que de equipamentos tecnológicos em si, e essa relação terapêutica e a habilidade humana influenciam no tratamento e auxiliam a melhorar os resultados obtidos. E, com a reorganização da rede de serviços não baseada na lógica hospitalocêntrica, novas atitudes da equipe são primordiais.

Os estudos aqui relacionados identificam o tema das tecnologias de saúde como algo importante, corroborando com a discussão brasileira e inglesa, por exemplo. Na verdade, sensibilizando profissionais de saúde e sociedade a respeito de ferramentas, para além das físicas, que são necessárias no cuidado do paciente psiquiátrico (as relacionais e afetivas) (PICKERSGILL, 2013), indo ao encontro do próximo tema a ser explorado.

\subsection{Institucionalização da loucura e suas consequências}

Falar em saúde mental hoje em dia, infelizmente, ainda nos remete à ideia dos manicômios, asilos e hospitais psiquiátricos. Mesmo que tantas mudanças políticas e assistenciais venham ocorrendo no Brasil e no mundo, a institucionalização das práticas clínicas nessa área ainda acontece e é discutida (AMARANTE, 2009).

O processo de institucionalização da loucura apareceu como ponto importante na discussão dos estudos, assim como suas consequências. Uma delas é a medicalização da loucura e a percepção de que toda experiência humana traz consigo sinais de adoecimento (PALOMO, 2013).

Cada vez mais, também a partir da publicação do Manual de Diagnóstico e Estatística dos Transtornos Mentais $5^{a}$ edição (DSM-V), os limites entre saúde e doença na contemporaneidade estão cada vez mais tênues. O contexto de vida deve ser um critério de avaliaçáo de todo e qualquer processo de adoecimento, caso contrário corre-se o risco de uma avaliação individual e pouco significativa. Aqui está a medicalização da vida.

Outra consequência é a perda de autonomia que a hospitalização faz as pessoas experimentarem, quando estão sujeitas aos cuidados de profissionais que tornam a rotina um procedimento (POLS, 2006), deixando o cotidiano fora dessa perspectiva. A individualidade é perdida quando pacientes e profissionais estão subordinados a um processo massificante de institucionalização.

Formas de cuidar evoluíram felizmente para fora das grandes instituiçôes. Claro que há momentos em que a hospitalização breve é necessária frente a diferentes psicopatologias. Mas há todo um esforço de desmantelamento desses equipamentos asilares e, em substituição, implantação de rede de serviços ambulatorial, territorial e comunitária. Afinal, o conhecimento evoluiu de forma a compreender que o cuidado próximo da família e da comunidade era mais benéfico para todos. Este aspecto aparece nas publicaçôes encontradas nesse levantamento.

Esta é uma discussão que traz consigo questôes como estigma e estereótipos que ainda estâo carregados de uma visão da doença mental de antigamente, daqueles presos em hospitais. Há que se difundir esse novo paradigma da saúde mental, e o campo CTS tem muito a contribuir nesse sentido.

\section{Conclusão}

Este ensaio permitiu que alguns periódicos com uma abordagem CTS fossem identificados, a partir de artigos publicados, com a temática da saúde mental. Para surpresa dos autores, foram localizadas algumas publicaçóes que realizavam esse entrelaçamento de temas, e a forma como foram discutidos internacionalmente não difere de maneira muito significativa de como pesquisadores e profissionais brasileiros o fazem nacionalmente.

Há uma estreita relação com temas discutidos no Brasil, como (des)institucionalização, a absorção do conhecimento produzido em saúde mental direto pela clínica praticada nos serviços, a estigmatização do problema mental ainda hoje e a grande lacuna existente entre prática e políticas públicas.

A forma como a construçáo de conhecimento para cuidado em saúde mental ocorre se dá muito a partir de relatos da prática junto aos usuários. E assim foram muitos os artigos aqui identificados. Com uma diferença evidente na forma de financiamento desses projetos, contando em grande parte com indústrias farmacêuticas.

Os temas discutidos neste ensaio tecem relação com a terapia ocupacional. Historicamente, a profissão vem acompanhando tais mudanças no campo do pensar, do atuar e do elaborar políticas públicas em saúde mental. Atuação esta que vem criticar práticas ainda manicomiais, mesmo em equipamentos substitutivos a tal lógica. A profissão, que tem como um de seus objetivos finais a inserção social dos sujeitos, dialoga de forma considerável com os resultados desta pesquisa.

Em nossas discussōes atuais, fica cada vez mais evidente que a tão sonhada abertura da "caixa-preta" sinalizada por Latour (2011) não pode ocorrer apenas pelas disciplinas, pois sua complexidade exige 
uma configuração articulada de saberes e práticas, profissionais e da sociedade, novas controvérsias que, por fim, mobilizam a elaboração de perguntas e suas possíveis explicaçôes. Influenciam, de fato, a forma como temos realizado o cuidado a essas pessoas e que saúde mental pode e deve estar na agenda CTS de diferentes países.

\section{Referências}

AMARANTE, P. Reforma psiquiátrica e epistemologia. Cadernos Brasileiros de Saúde Mental, Florianópolis, v. 1, n. 1, p. 1-7, 2009.

ARMSTRONG, D. Origins of the problem of health-related behaviours: a genealogical study. Social Studies of Science, Thousand Oaks, v. 39, n. 6, p. 910-926, 2009.

BARDIN, L. Análise de conteúdo. Lisboa: Ediçōes 70, 2009.

BRASIL. Ministério da Saúde. Secretaria de Ciência, Tecnologia e insumos estratégicos. Departamento de Ciência e Tecnologia. Agenda nacional de prioridades de pesquisa em saúde. Brasília: Editora do Ministério da Saúde, 2008.

IGLESIAS, A.; AVELLAR, L. Z. Apoio Matricial: um estudo bibliográfico. Ciência \& Saúde Coletiva, Rio de Janeiro, v. 19, n. 9, p. 3791-3798, 2014.

KENDALL-TAYLOR, N. Conflicting models of mind: mapping the gaps between expert and public understandings of child mental health. Science Communication, Thousand Oaks, v. 34, n. 6, p. 695-726, 2012.

LATOUR, B. Ciência em ação: como seguir cientistas e engenheiros sociedade afora. São Paulo: Editora da UNESP, 2011.

LEWISON, G. et al. Outputs and expenditures on health research in eight disease areas using a bibliometric approach, 1996-2001. Research Evaluation, Oxford, v. 13, n. 3, p. 181-188, 2004.

MENDES, K. D. S.; SILVEIRA, R. C. C. P.; GALVÃO, C. M. Revisão integrativa: método de pesquisa para a incorporação de evidências na saúde e na enfermagem.
Texto \& Contexto Enfermagem, Florianópolis, v. 17, n. 4, p. 758-64, 2008.

MERHY, E. E. Um ensaio sobre o médico e suas valises tecnológicas: contribuiçōes para compreender as reestruturaçôes produtivas do setor Saúde. Interface - Comunicação, Saúde, Educação, Botucatu, v. 4, n. 6, p. 109-116, 2000.

ORGANIZAÇÃO MUNDIAL DA SAÚDE - OMS. Relatório mundial de saúde: saúde mental: nova concepção, nova esperança. Genebra: OMS, 2002. Disponível em: <http://www.who.int/whr/2001/en/whr01_ po.pdf>. Acesso em: 12 out. 2014.

PALOMO, J. L. R. A. Locos entre cuerdos, cuerdos en ambientes patológicos. Arbor, Madrid, v. 189, n. 763, a069, 2013.

PICKERSGILL, M. How personality became treatable: the mutual constitution of clinical knowledge and mental health law. Social Studies of Science, Thousand Oaks, v. 43, n. 30, p. 1-24, 2013.

PINTO, M. C. L. R. O enfermeiro como facilitador de processos de educação permanente em saúde: fronteiras e perspectivas da atuação profissional. 2011. $170 \mathrm{f}$. Dissertação (Mestrado em Enfermagem) - Universidade Federal de São Carlos, São Carlos, 2012.

POLS, J. Accounting and washing: good care in long-term psychiatry. Science Technology Human Values, Thousand Oaks, v. 31, n. 4, p. 409-430, 2006.

SANTOS, B. S. Um discurso sobre as ciências. Porto: Edições Afrontamento, 1987.

THORNICROFT, G.; TANSELLA, M. Boas práticas em saúde mental comunitária. Barueri: Manole, 2010.

WEBER, M. A.; BACKER, T. E. Science-based communication strategy for a federal health agency. Science Communication, Thousand Oaks, v. 35, n.5, p. 1-11, 2013.

ZAUITH, G.; OGATA, M. N.; HAYASHI, M. C. P. I. Um breve panorama sobre a educaçáo CTS no Brasil. In: HOFFMANN, W. A. M. (Ed.). Ciência, tecnologia e sociedade: desafios da construçáo do conhecimento. São Carlos: EdUFSCar, 2011. p. 21-38.

\section{Contribuição dos Autores}

Alana de Paiva Nogueira Fornereto Gozzi: Concepção e elaboração do texto, revisão bibliográfica, organização de fontes, coleta e análise dos dados, redação do texto e sua finalização. Edson Malvezzi, Meliza Cristina da Silva e Márcia Niituma Ogata: Concepção do texto, revisão, organização do texto e sua finalização. Todos os autores aprovaram a versão final do texto. 